\title{
On the Chern Number of a Filtration
}

\author{
M. E. Rossi (*) - G. Valla $(*)$
}

\section{Introduction.}

The Hilbert function of a local ring $(A, \mathfrak{m})$ is the function which associates to each non negative integer $n$ the minimal number of generators of $\mathfrak{m}^{n}$. It gives deep information on the corresponding singularity and, owing to its geometric significance, has been studied extensively.

In the case of a standard graded algebra, the Hilbert function is well understood, at least in the Cohen-Macaulay case. Instead, very little is known in the local case and we do not have a guess of the shape of the Hilbert function, even in the case of a Cohen-Macalay one dimensional domain.

Due to this lack of information, a long list of papers have been written where constraints on the possible Hilbert functions of a Cohen-Macaulay local ring are described. Usually, restrictions have been found on what are called the Hilbert coefficients of the maximal ideal $\mathfrak{m}$ of $A$.

The first coefficient, $e_{0}(\mathfrak{m})$, is called the multiplicity of $\mathfrak{m}$ and, due to its geometric meaning, has been studied very deeply, see for example [11]. The other coefficients are not as well understood, either geometrically or in terms of how they are related to algebraic properties of the ideal or ring.

The goal of this paper is the study of the second (after the multiplicity) coefficient of the Hilbert polynomial of the local ring $(A, \mathfrak{m})$. This integer, $e_{1}$, has been recently interpreted in [9] as a tracking number of the Rees algebra of $A$ in the set of all such algebras with the same multiplicity.

(*) Indirizzo degli A.: Dipartimento di Matematica, Universitá di Genova, Via Dodecaneso 35, I-16146 Genova, Italy.

E-mail: rossim@dima.unige.it valla@dima.unige.it

Research partially supported by the "Ministero dell'Universitá e della Ricerca Scientifica" in the framework of the National Research Network (PRIN 2005) "Algebra commutativa, combinatorica e computazionale".

2000 Mathematics Subject Classification. Primary 13H10; Secondary 13H15. 
Under various circumstances, it is also called the Chern number or coefficient of the local ring $A$. An interesting list of questions and conjectural statements about the values of $e_{1}$ for filtrations associated to an m-primary ideal of a local ring $A$ had been presented in a recent paper by W. Vasconcelos (see [18]).

In the Cohen-Macaulay case, starting from the work of D.G. Northeott in the 60 's, several results have been proved which give some relationships between the Hilbert coefficients, thus implying some constrains on the Hilbert function. The way was enlightened by Northeott who proved that, if $(A, \mathfrak{m})$ is a Cohen-Macaulay local ring, then

$$
e_{1}(\mathfrak{m}) \geq e_{0}(\mathfrak{m})-1 \text {. }
$$

Thus, for example, the series $\frac{1+2 z-z^{2}}{1-z}$ cannot be the Hilbert series of a Cohen-Macaulay local ring of dimension one because $e_{1}(\mathfrak{m})=0$, while $e_{0}(\mathfrak{m})=2$.

Several results along this line has been proved in the last years, often extending the framework to Hilbert function associated to a filtration, but always assuming the Cohen-Macaulayness of the basic ring. For a large overview of these kind of results one can see [10].

More recently Goto and Nishida in [5] were able to extend Northcott bound to the case of a primary ideal, avoiding, for the first time in this setting, the assumption that the ring is Cohen-Macayulay. Of course, they need to introduce a correction term which vanishes in the Cohen-Macaulay case.

On the base of this result, Corso in [3] could handle in this wild generality a stronger upper bound for $e_{1}$ given by Elias and Valla in [4].

Here we will focus, instead, on an upper bound of $e_{1}$ which was introduced and studied by Huckaba and Marley in [7]. If $q$ is a primary ideal in the local Cohen-Macaulay ring $A$ and $J$ is an ideal generated by a maximal superficial sequence for $\mathfrak{q}$, then Valla proved in [16] that

$$
e_{0}(\mathfrak{q})=(1-d) \lambda(A / \mathfrak{q})+\lambda\left(\mathfrak{q} / \mathfrak{q}^{2}\right)+\lambda\left(\mathfrak{q}^{2} / J \mathfrak{q}\right),
$$

thus extending a classical result of Abhyankar on the maximal ideal to the m-primary case. The above formula shows that $\lambda\left(\mathfrak{q}^{2} / J \mathfrak{q}\right)$ does not depend on $J$ and, for the first time, properties of the Hilbert coefficients are related to superficial sequences. Since then, it was natural to consider the integers

$$
v_{j}(\mathfrak{q}):=\lambda\left(\mathfrak{q}^{j+1} / J \mathfrak{q}^{j}\right)
$$

and to investigate how they are involved in the study of the Hilbert series of 
a primary ideal. This has been done by Huckaba and Marley in [7] where they proved that for any ideal filtration $\mathcal{F}=\left\{I_{j}\right\}_{j \geq 0}$ of a Cohen-Macaulay local ring $A$, one has

$$
e_{1}(\mathcal{F}) \leq \sum_{j \geq 0} v_{j}(\mathcal{F})
$$

with equality if and only if the depth of the associated graded ring is at least $d-1$, if and only if the Hilbert series is

$$
P_{\mathcal{F}}(z)=\frac{\lambda\left(A / I_{0}\right)+\sum_{j \geq 0}\left(v_{j}(\mathcal{F})-v_{j+1}(\mathcal{F})\right) z^{j+1}}{(1-z)^{d}} .
$$

The first main result of this paper, Theorem 2.11, shows that given any good q-filtration MI of a module $M$ which is not necessarily Cohen-Macaulay, we have

$$
e_{1}(\mathbb{M I})-e_{1}(\mathbb{N}) \leq \sum_{j \geq 0} v_{j}(\mathbb{M})
$$

where $\mathbb{N}$ is the $J$-adic filtration on $M$. It is easy to see that, if $M$ is CohenMacaulay, then $e_{i}(\mathbb{N})=0$ for every $i \geq 1$, so that these integers are good correction terms when the Cohen-Macaulay assumption does not hold.

We are able to understand in Theorem 2.13 when equality holds above, at least for the case when $\mathbb{M}$ is the m-adic filtration on the local ring $A$. Surprisingly enough, we show that the equality $e_{1}(\mathfrak{m})-e_{1}(\mathbb{N})=\sum_{j \geq 0} v_{j}(\mathfrak{m})$ forces the ring $A$ to be Cohen-Macaulay and hence the associated graded ring to have almost maximal depth.

Thus we get the Cohen-Macaulayness of the ring $A$ as a consequence of the extremal behavior of the integer $e_{1}(\mathfrak{m})$. The result can be considered a confirm of the general philosophy of the paper of W. Vasconcelos [18] where the Chern number is conjectured to be a measure of the distance from the Cohen-Macaulyness of $A$.

This main result of the paper is a consequence of a nice and perhaps unexpected property of superficial elements which we prove in Lemma 2.1. It is essentially a kind of "Sally machine" for local rings.

In the last section we describe an application of these results, concerning an upper bound on the multiplicity of the Sally module of a good filtration of a module which is not necessarily Cohen-Macaulay. The bound is given in term of the same ingredients used by Huckaba-Marley for $e_{1}$. It is an extension to the non Cohen-Macaulay case of a result of Vaz Pinto in [19]. 


\section{An upper bound for the Chern number.}

Let $(A, \mathfrak{m})$ be a local ring and $\mathfrak{q}$ a primary ideal for $\mathfrak{m}$. If $M \neq\{0\}$ is a finitely generated $A$-module, a q-filtration on $M$ is a chain

$$
\mathbb{M}: \quad M=M_{0} \supseteq M_{1} \supseteq \cdots \supseteq M_{j} \supseteq \cdots
$$

such that $\mathfrak{q} M_{j} \subseteq M_{j+1}$ for every $j$. In the case $M_{j+1}=\mathfrak{q} M_{j}$ for $j \gg 0$, we say that the filtration $\mathbb{M}$ is a good $q$-filtration.

If $N$ is a submodule of $M$, it is clear that $\left\{\left(N+M_{j}\right) / N\right\}_{j \geq 0}$ is a good $\mathfrak{q}$ filtration of $M / N$ which we denote by $M / N$.

Given the good $\mathfrak{q}$-filtration $\mathbb{M}$ on $M$ we let $g r_{\mathfrak{q}}(A):=\bigoplus_{j \geq 0} \mathfrak{q}^{j} / \mathfrak{q}^{j+1}$, $g r_{\mathrm{M}}(M):=\bigoplus_{j \geq 0} M_{j} / M_{j+1}$. We know that $g r_{\mathrm{MI}}(M)$ is a graded $g r_{\mathfrak{q}}(\dot{j} \bar{A})$-module; further each element $a \in A$ has a natural image $a^{*}$ in $g r_{\mathfrak{q}}(A)$ which is 0 if $a=0$, is $a^{*}=\bar{a} \in \mathfrak{q}^{t} / \mathfrak{q}^{t+1}$ if $a \in \mathfrak{q}^{t} \backslash \mathfrak{q}^{t+1}$.

A tool which has been very useful in the study of the depth of blowingup rings is the so called Valla-Valabrega criterion (see [15]). Given the ideal $I=\left(a_{1}, \ldots, a_{r}\right)$ in $A$, the elements $a_{1}^{*}, \ldots, a_{r}^{*}$ form a regular sequence on $g r_{\mathrm{MI}}(M)$ if and only if they form a regular sequence on $M$ and $I M \cap M_{j}=$ $=I M_{j-1}$ for every $j \geq 1$.

We recall that an element $a \in \mathfrak{q}$ is called $\mathbb{N}$-superficial for $\mathfrak{q}$ if there exists a non-negative integer $c$ such that $\left(M_{j+1}:_{M} a\right) \cap M_{c}=M_{j}$ for every $j \geq c$. If we assume that $M$ has positive dimension, then every superficial element $a$ for $\mathfrak{q}$ has order one, that is $a \in \mathfrak{q} \backslash \mathfrak{q}^{2}$. Further, since we are assuming that the residue field $A / \mathfrak{m}$ is infinite, it is well known that superficial elements do exist.

A sequence of elements $a_{1}, \ldots, a_{r}$ will be called a M-superficial sequence for $\mathfrak{q}$ if for every $j=1, \ldots, r$ the element $a_{j}$ is an (M/ $\left./\left(a_{1}, \ldots, a_{j-1}\right) M\right)$-superficial element for $\mathfrak{q}$. It is well known that if $a_{1}, \ldots, a_{r}$ is a $\mathbb{N}$-superficial sequence for $\mathfrak{q}$ and $J$ is the ideal they generate, then we have the following properties which show the relevance of superficial sequences in the study of the depth of blowing-up rings.

(1) $\left\{a_{1}, \ldots, a_{r}\right\}$ is a regular sequence on $M$ if and only if depth $M \geq r$.

(2) $\left\{a_{1}^{*}, \ldots, a_{r}^{*}\right\}$ is a regular sequence on $g r_{\mathrm{M}}(M)$

if and only if depth $g r_{\mathbb{Y}}(M) \geq r$.

If the sequence is maximal then, as in [14],

$$
M_{n+1}=J M_{n} \text { for } n \gg 0 .
$$


Hence $\mathbb{M}$ is a good $J$-filtration. Further by 8.3.5 in [8] , $J$ is minimal with respect to this property. It follows that if $x_{1}, \ldots, x_{d} \in J$ is a maximal Msuperficial sequence, then $J=\left(x_{1}, \ldots, x_{d}\right)$.

We recall now that the Hilbert function of the filtration $M$ is the function

$$
H_{\mathbb{M}}(j):=\lambda\left(M_{j} / M_{j+1}\right) .
$$

The Hilbert series of M is

$$
P_{\mathrm{M}}(z):=\sum_{j \geq 0} H_{\mathrm{M}}(j) z^{j}=\frac{h_{\mathrm{M}}(z)}{(1-z)^{d}}
$$

where $d$ is the dimension of $M$. For every $i \geq 0$ we let $e_{i}(\mathrm{MI}):=\frac{h_{\mathrm{MI}}^{(i)}(1)}{i !}$; then for every $j \gg 0$ we have $H_{\mathbb{M}}(j)=p_{\mathbb{M}}(j)$ where

$$
p_{\mathbb{M}}(X):=\sum_{i=0}^{d-1}(-1)^{i} e_{i}(\mathbb{M})\left(\begin{array}{c}
X+d-i-1 \\
d-i-1
\end{array}\right)
$$

is a polynomial with rational coefficients and degree $d-1$ which is called the Hilbert polynomial of M. Its coefficients $e_{i}(\mathrm{MI})$ are the Hilbert coefficients of $\mathrm{M}$.

It is also relevant to consider the numerical function

$$
H_{\mathrm{M}}^{1}(j):=\lambda\left(M / M_{j+1}\right)=\sum_{i=0}^{j} H_{\mathbb{M}}(i)
$$

which is called the Hilbert-Samuel function of the filtered module $M$. Its generating function is simply the series

$$
P_{\mathrm{MI}}^{1}(z)=\frac{P_{\mathrm{M}}(z)}{(1-z)}=\frac{h_{\mathrm{MI}}(z)}{(1-z)^{d+1}} .
$$

It is clear that the polynomial

$$
p_{\mathrm{MI}}^{1}(X):=\sum_{i=0}^{d}(-1)^{i} e_{i}(\mathrm{M})\left(\begin{array}{c}
X+d-i \\
d-i
\end{array}\right)
$$

verifies the equality $p_{\mathbb{M}}^{1}(j)=H_{\mathbb{M}}^{1}(j)$ for $j \gg 0$. It is called the Hilbert-Samuel polynomial of $\mathrm{M}$.

The first Hilbert coefficient $e_{0}$ is the multiplicity of $\mathbb{M}$; by Proposition 11.4 in [2] we know that

$$
e_{0}(\mathbb{N})=e_{0}(\mathbb{N})
$$


for every couple of good $\mathfrak{q}$-filtrations. Also, if $M$ is artinian, then $e_{0}(\mathbb{M})=\lambda(M)$.

The classical case is when the module $M$ is the ring $A$ and the filtration is given by the powers of a primary ideal $I$ of $A$. This is called the $I$-adic filtration on $A$ and the corresponding Hilbert coefficients are simply indicated by $e_{i}(I)$.

For every $a \in \mathfrak{q}$ one can prove that

$$
P_{\mathrm{MI}}(z) \leq P_{\mathrm{M} / a M}^{1}(z)
$$

(see [10] and [12]).

As a consequence of (5), we get some useful properties of superficial elements. In the following $a$ will be a MI-superficial element for $\mathfrak{q}$ and $d$ the dimension of $M$. We have

$$
\begin{gathered}
\operatorname{dim}(M / a M)=d-1 \\
e_{j}(\mathrm{M})=e_{j}(\mathrm{M} / a M) \text { for every } j=0, \ldots, d-2 . \\
e_{d-1}(\mathrm{M} / a M)=e_{d-1}(\mathrm{M})+(-1)^{d-1} \lambda\left(0:_{M} a\right) .
\end{gathered}
$$

We will also need a property of superficial elements which seems to be neglected in the literature. Recall that if $a$ is $M$-regular, then $M / a M$ is Cohen-Macaulay if and only if $M$ is Cohen-Macaulay. We prove, as a consequence of the following Lemma, that the same holds for a superficial element, if the dimension of the module $M$ is at least two.

In the following we denote by $H_{\mathrm{q}}^{i}(M)$ the $i$-th local cohomology module of $M$ with respect to $\mathfrak{q}$. We know that $H_{\mathfrak{q}}^{0}(M):=\bigcup_{j \geq 0}\left(0:_{M} \mathfrak{q}^{j}\right)=0:_{M} \mathfrak{q}^{t}$ for every $t \gg 0$ and $\min \left\{i \mid H_{\mathfrak{q}}^{i}(M) \neq 0\right\}=\operatorname{grade}(\mathfrak{q}, M)$, where grade $(\mathfrak{q}, M)$ is the common cardinality of all the maximal $M$-regular sequences of elements in $\mathfrak{q}$.

Lemma 2.1. Let $\mathbb{M}$ be a good $\mathfrak{q}$-filtration of a module $M$, a an M-superficial element for $\mathfrak{q}$ and $j \geq 1$. Then $\operatorname{grade}(\mathfrak{q}, M) \geq j+1$ if and only if $\operatorname{grade}(\mathfrak{q}, M / a M) \geq j$.

Proof. Let grade $(\mathfrak{q}, M) \geq j+1$; then depth $M>0$ so that $a$ is $M$ regular. This implies grade $(\mathfrak{q}, M / a M)=\operatorname{grade}(\mathfrak{q}, M)-1 \geq j+1-1=j$.

Let us assume now that grade( $\mathfrak{q}, M / a M) \geq j$. Since $j \geq 1$, this implies $H_{\mathfrak{q}}^{0}(M / a M)=0$. Hence $H_{\mathfrak{q}}^{0}(a M)=H_{\mathfrak{q}}^{0}(M)$, so that $H_{\mathfrak{q}}^{0}(M) \subseteq a M$. We claim that $H_{\mathfrak{q}}^{0}(M)=a H_{\mathfrak{q}}^{0}(M)$. If this is the case, then, by Nakayama, we get 
$H_{\mathfrak{q}}^{0}(M)=0$ which implies depth $M>0$, so that $a$ is $M$-regular. Hence

$$
\operatorname{grade}(\mathfrak{q}, M)=\operatorname{grade}(\mathfrak{q}, M / a M)+1 \geq j+1,
$$

as wanted.

Let us prove the claim. Suppose by contradiction that

$$
a H_{\mathfrak{q}}^{0}(M) \subsetneq H_{\mathfrak{q}}^{0}(M) \subseteq a M,
$$

and let $a x \in H_{\mathfrak{q}}^{0}(M), x \in M \backslash H_{\mathfrak{q}}^{0}(M)$. This means that for every $t \gg 0$ we have

$$
\left\{\begin{array}{l}
a \mathfrak{q}^{t} x=0 \\
\mathfrak{q}^{t} x \neq 0
\end{array} .\right.
$$

We prove that this implies that $\alpha$ is not $\mathbb{M}$-superficial for $\mathfrak{q}$. Namely, given a positive integer $c$, we can find an integer $t \geq c$ and an element $d \in \mathfrak{q}^{t}$ such that $a d x=0$ and $d x \neq 0$. Since $\cap M_{i}=\{0\}$, we have $d x \in M_{j-1} \backslash M_{j}$ for some integer $j$. Now, $d \in \mathfrak{q}^{t}$ hence $d x \in M_{t} \subseteq M_{c}$, which implies $j \geq c$. Finally we have $d x \in M_{c}, d x \notin M_{j}$ and $a d x=0 \in M_{j+1}$, hence

$$
\left(M_{j+1}: M a\right) \cap M_{c} \ngtr M_{j} .
$$

The claim and the Lemma are proved.

In establishing the properties of the Hilbert coefficients of a filtered module $M$, it will be convenient to use induction on the dimension of the module. To start the induction we need first to study the one-dimensional case.

Let us be given a good $\mathfrak{q}$-filtration $\mathrm{MI}=\left\{M_{j}\right\}_{j \geq 0}$ on a module $M$ of dimension 1. We have $H_{\mathbb{M}}(n)=p_{\mathrm{MI}}(n)=e_{0}(\mathbb{M})$ for $n \gg 0$ so that we define for every $j$

$$
u_{j}(\mathbb{M}):=e_{0}(\mathbb{M})-H_{\mathbb{M}}(j)
$$

Lemma 2.2. Let $\mathbb{M}$ be a good $\mathfrak{q}$-filtration of a module $M$ of dimension one. If $a$ is an M-superficial element for $\mathfrak{q}$, then for every $j \geq 0$ we have

$$
u_{j}(\mathbb{M})=\lambda\left(M_{j+1} / a M_{j}\right)-\lambda\left(0:_{M_{j}} a\right)
$$

Proof. By (6) we have

$$
\begin{aligned}
e_{0}(\mathbb{M}) & =e_{0}(\mathbb{M} / a M)-\lambda\left(0:_{M} a\right)=\lambda(M / a M)-\lambda\left(0:_{M} a\right) \\
& =\lambda\left(M / a M_{j}\right)-\lambda\left(a M / a M_{j}\right)-\lambda\left(0:_{M} a\right) .
\end{aligned}
$$


By using the following exact sequence

$$
0 \rightarrow\left(0:_{M} a\right) /\left(0:_{M_{j}} a\right) \rightarrow M / M_{j} \rightarrow a M / a M_{j} \rightarrow 0
$$

we get

$$
e_{0}(\mathbb{M})=\lambda\left(M / a M_{j}\right)-\lambda\left(M / M_{j}\right)+\lambda\left(\left(0:_{M} a\right) /\left(0:_{M_{j}} a\right)\right)-\lambda\left(0:_{M} a\right)
$$

and finally

$$
\begin{aligned}
u_{j}(\mathbb{M}) & =e_{0}(\mathbb{M})-\lambda\left(M_{j} / M_{j+1}\right) \\
& =\lambda\left(M / a M_{j}\right)-\lambda\left(M / M_{j}\right)-\lambda\left(0:_{M_{j}} a\right)-\lambda\left(M_{j} / M_{j+1}\right) \\
& =\lambda\left(M_{j+1} / a M_{j}\right)-\lambda\left(0:_{M_{j}} a\right) .
\end{aligned}
$$

It follows that, in the case $M$ is one dimensional and Cohen-Macaulay, then $u_{j}(\mathbb{M})=\lambda\left(M_{j+1} / a M_{j}\right)$ is non negative and we have, for every $j \geq 0$, $H_{\mathrm{M}}(j)=e_{0}(\mathbb{M})-\lambda\left(M_{j+1} / a M_{j}\right) \leq e_{0}(\mathbb{M})$.

It will be useful to write down the Hilbert coefficients through the integers $u_{j}(\mathbb{M})$.

LEMMA 2.3. Let $\mathbb{M}$ be a good q-filtration of a module $M$ of dimension one. Then for every $j \geq 0$ we have

$$
e_{j}(\mathbb{M I})=\sum_{k \geq j-1}\left(\begin{array}{c}
k \\
j-1
\end{array}\right) u_{k}(\mathbb{M}) .
$$

Proof. We have

$$
P_{\mathrm{MI}}(z)=\frac{h_{\mathrm{MI}}(z)}{1-z}=\sum_{j \geq 0} H_{\mathrm{MI}}(j) z^{j} .
$$

Hence, if we write $h_{\mathrm{MI}}(z)=h_{0}(\mathrm{MI})+h_{1}(\mathbb{M}) z+\cdots+h_{s}(\mathbb{M}) z^{s}$, then we get for every $k \geq 1$

$$
h_{k}(\mathbb{M})=H_{\mathbb{M}}(k)-H_{\mathbb{M}}(k-1)=u_{k-1}(\mathbb{M})-u_{k}(\mathbb{M}) .
$$

Finally

$$
\begin{aligned}
e_{j}(\mathbb{M}) & =\frac{h_{\mathbb{N}}^{(j)}(1)}{j !}=\sum_{k \geq j}\left(\begin{array}{l}
k \\
j
\end{array}\right) h_{k}(\mathbb{M})=\sum_{k \geq j}\left(\begin{array}{l}
k \\
j
\end{array}\right)\left(u_{k-1}(\mathbb{M I})-u_{k}(\mathbb{M I})\right) \\
& =\sum_{k \geq j-1}\left(\begin{array}{c}
k \\
j-1
\end{array}\right) u_{k}(\mathbb{M}) .
\end{aligned}
$$


If we apply the above Lemma in the case $M$ is Cohen-Macaulay, and using the fact that the integers $u_{k}(\mathbb{M})$ are non negative, we get

$$
e_{1}(\mathbb{M})=\sum_{k \geq 0} u_{k}(\mathbb{M}) \geq u_{0}(\mathbb{N})+u_{1}(\mathbb{M}) \geq u_{0}(\mathbb{N}) .
$$

Since we have $u_{0}(\mathbb{M})=e_{0}(\mathbb{M})-\lambda\left(M / M_{1}\right)$, and $u_{0}(\mathbb{M})+u_{1}(\mathbb{M})=$ $=2 e_{0}(\mathbb{M})-\lambda\left(M / M_{2}\right)$, we trivially get

$$
e_{1}(\mathbb{M}) \geq e_{0}(\mathbb{M})-\lambda\left(M / M_{1}\right)
$$

and

$$
e_{1}(\mathbb{M}) \geq 2 e_{0}(\mathbb{M})-\lambda\left(M / M_{2}\right),
$$

which are the bounds by Northcott and Elias-Valla, in the one dimensional Cohen-Macaulay case.

If we do not assume that $M$ is Cohen-Macaulay, the integers $u_{k}(\mathrm{~N} I)$ can be negative and the above formulas do not hold anymore.

At this point we are going to describe the correction term which will appear in our upper bound of $e_{1}$.

Given a good $\mathfrak{q}$-filtration $\mathrm{M}$ of the module $M$ of dimension $d$, let $a_{1}, \ldots, a_{d}$ be a M-superficial sequence for $\mathfrak{q}$. We denote by $J$ the ideal they generate and consider the $J$-adic filtration of the module $M$. This is by definition the filtration

$$
\mathbb{N}:=\left\{J^{j} M\right\}_{j \geq 0}
$$

which is clearly a good $J$-filtration. By (3) M is also a good $J$-filtration, so that, by (4), $e_{0}(\mathbb{M})=e_{0}(\mathbb{N})$.

In the case $M$ is Cohen-Macaulay, the elements $a_{1}, \ldots, a_{d}$ form a regular sequence on $M$ so that $J^{i} M / J^{i+1} M \simeq(M / J M)^{\left(\begin{array}{c}d+i-1 \\ i\end{array}\right) \text {. This implies that }}$ the Hilbert Series of $\mathbb{N}$ is $P_{\mathrm{N}}(z)=\frac{\lambda(M / J M)}{(1-z)^{d}}$ and thus $e_{i}(\mathbb{N})=0$ for every $i \geq 1$. This proves that these integers give a good measure of how $M$ differs from being Cohen-Macaulay. In the case $M=A$, Vasconcelos in [17] and [18] conjectured that if $A$ is not Cohen-Macaulay, then $e_{1}(J)<0$ and he proved it for large classes of local rings.

First we prove that $e_{1}(\mathbb{N}) \leq 0$ in the one dimensional case where the integer $e_{1}(\mathbb{N})$ can be easily related with the 0 -th local cohomology module of $M$.

Lemma 2.4. Let M be a good q-filtration of a module $M$ of dimension one. If a is an $\mathbb{M}$-superficial element for $\mathfrak{q}$, then for every $t \gg 0$ we have

$$
e_{1}(\mathbb{N})=-\lambda\left(0: M a^{t}\right) .
$$


Proof. We have $p_{\mathbb{N}}^{1}(X)=e_{0}(\mathbb{N})(X+1)-e_{1}(\mathbb{N})=e_{0}(\mathbb{N})(X+1)-$ $-e_{1}(\mathbb{N})$. On the other hand we have short exact sequences

$$
\begin{gathered}
0 \rightarrow\left(0:_{M} a^{k}\right) / 0: a M a^{k} \rightarrow M / a M \stackrel{a}{\rightarrow} a^{k} M / a^{k+1} M \rightarrow 0 \\
0 \rightarrow 0: M a \rightarrow 0:_{M} a^{i+1} \stackrel{a}{\rightarrow} 0:_{a M} a^{i} \rightarrow 0
\end{gathered}
$$

which give

$$
\begin{aligned}
& H_{\mathbb{N}}^{1}(j)=\sum_{i=0}^{j} H_{\mathbb{N}}(i)=\sum_{i=0}^{j} \lambda\left(a^{i} M / a^{i+1} M\right)=\sum_{i=0}^{j}\left[\lambda(M / a M)-\lambda\left(0:_{M} a^{i} / 0:_{a M} a^{i}\right)\right] \\
& =(j+1) \lambda(M / a M)-\lambda\left(0:_{M} a\right)-\sum_{i=1}^{j}\left[\lambda\left(0:_{M} a^{i+1}\right)-\lambda\left(0:_{a M} a^{i}\right)\right]+\lambda\left(0:_{M} a^{j+1}\right) \\
& =(j+1)\left[\lambda(M / a M)-\lambda\left(0:_{M} a\right)\right]+\lambda\left(0:_{M} a^{j+1}\right)=(j+1) e_{0}(\mathbb{M})+\lambda\left(0:_{M} a^{j+1}\right) .
\end{aligned}
$$

The conclusion follows.

In this section we denote by $W$ the 0 -th local cohomology module $H_{\mathfrak{m}}^{0}(M)$ of $M$ with respect to $\mathrm{m}$. Recall that

$$
W:=\bigcup_{j \geq 0}\left(0: M \mathfrak{m}^{j}\right)
$$

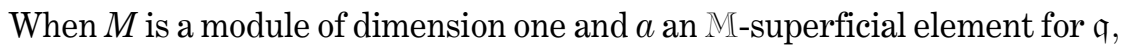
then $M / a M$ has finite length so that $0:_{A}(M / a M)$ is a primary ideal. Now it is easy to see that $0:_{A}(M / a M) \subseteq \sqrt{(a)+0:_{A} M}$, and thus $(a)+0:_{A} M$ is a primary ideal too. This means that a power of $m$ is contained in $(a)+0:_{A} M$, say $\mathfrak{m}^{s} \subseteq(a)+0:_{A} M$. Hence, for $t \gg 0$

$$
W=0::_{M} \mathfrak{m}^{t} \subseteq 0::_{M} a^{t} \subseteq 0::_{M} \mathfrak{m}^{t s}=W .
$$

The above lemma and this remark give

$$
\lambda(W)=-e_{1}(\mathbb{N})
$$

as was already proved in [5], Lemma 2.4.

Given a good q-filtration of the module $M$, we consider now the corresponding filtration of the saturated module $M^{\text {sat }}:=M / W$. This is the filtration

$$
\mathbb{M}^{\text {sat }}:=\mathbb{M} / W=\left\{M_{n}+W / W\right\}_{n \geq 0}
$$

Since $W$ has finite length and $\cap M_{i}=\{0\}$, we have $M_{i} \cap W=\{0\}$ for every $i \gg 0$. This implies $p_{\mathbb{Y}}(X)=p_{\mathbb{M}^{s a t}}(X)$.

Further, it is clear that for every $j \geq 0$ we have an exact sequence

$$
0 \rightarrow W /\left(M_{j+1} \cap W\right) \rightarrow M / M_{j+1} \rightarrow M /\left(M_{j+1}+W\right) \rightarrow 0
$$


so that for every $j \gg 0$ we have

$$
\lambda\left(M / M_{j+1}\right)=\lambda\left[M /\left(M_{j+1}+W\right)\right]+\lambda(W)
$$

which implies

$$
p_{\mathrm{MI}}^{1}(X)=p_{\mathbb{M}^{\text {sat }}}^{1}(X)+\lambda(W) .
$$

This proves the following result:

Proposition 2.5. Let M be a good a-filtration of the module $M$ and $W:=H_{\mathfrak{m}}^{0}(M)$. If we let $d:=\operatorname{dim}(M)$ and $\mathbb{M}^{\text {sat }}:=\mathbb{M} / W$, then

$$
e_{i}(\mathbb{M})=e_{i}\left(\mathbb{M}^{s a t}\right) \quad 0 \leq i \leq d-1, \quad e_{d}(\mathbb{M})=e_{d}\left(\mathbb{M}^{s a t}\right)+(-1)^{d} \lambda(W) .
$$

We remark that, if $\operatorname{dim}(M) \geq 1$, the module $M / W$ has always positive depth. This is the reason why, sometimes, we move our attention from the module $M$ to the module $M / W$. This will be the strategy of the proof of the next proposition which gives, in the one dimensional case, the promised upper bound for $e_{1}$.

Proposition 2.6. Let M be a good q-filtration of a module $M$ of dimension one. If a is a $\mathbb{M}$-superficial element for q and $\mathbb{N}$ the (a)-adic filtration on $M$, then

$$
e_{1}(\mathbb{M})-e_{1}(\mathbb{N}) \leq \sum_{j \geq 0} \lambda\left(M_{j+1} / a M_{j}\right)
$$

If $W \subseteq M_{1}$ and equality holds above, then $M$ is Cohen-Macaulay.

Proof. By Proposition 2.5 and (10) we have

$$
e_{1}(\mathbb{M})=e_{1}\left(\mathbb{M}^{s a t}\right)-\lambda(W)=e_{1}\left(\mathbb{M}^{s a t}\right)+e_{1}(\mathbb{N})
$$

so that we need to prove that $e_{1}\left(\mathbb{M}^{\text {sat }}\right) \leq \sum_{j \geq 0} \lambda\left(M_{j+1} / a M_{j}\right)$.

Now $M / W$ is Cohen-Macaulay and $a$ is regular on $M / W$, hence by Lemma 2.3 and Lemma 2.2, we get

$$
\begin{aligned}
e_{1}\left(\mathbb{M}^{s a t}\right) & =\sum_{j \geq 0} u_{j}\left(\mathbb{M}^{s a t}\right)=\sum_{j \geq 0} \lambda\left(M_{j+1}^{s a t} / a M_{j}^{s a t}\right) \\
& =\sum_{j \geq 0} \lambda\left[\frac{M_{j+1}+W}{a M_{j}+W}\right]=\sum_{j \geq 0} \lambda\left[\frac{M_{j+1}}{a M_{j}+M_{j+1} \cap W}\right] \\
& \leq \sum_{j \geq 0} \lambda\left(M_{j+1} / a M_{j}\right) .
\end{aligned}
$$


The first assertion follows. In particular equality holds if and only if $M_{j+1} \cap W \subseteq a M_{j}$ for every $j \geq 0$. Let as assume $W \subseteq M_{1}$ and equality above; then we have $W=W \cap M_{1} \subseteq a M$. Now recall that $W=0:_{M} a^{t}$ for $t \gg 0$, hence if $c \in W$ then $c=a m$ with $a^{t} c=a^{t+1} m=0$. This implies $m \in 0: M a^{t+1}=W$ so that $W \subseteq a W$ and, by Nakayama, $W=0$.

In the last section we need to compare the Hilbert coefficients of the $J$ adic filtration $\mathbb{N}$ on $M$ with those of the following filtration. Given a good $\mathfrak{q}-$ filtration $\mathbb{M}$ on $M$ and the ideal $J$ generated by a maximal $\mathbb{M}$-superficial sequence for $\mathfrak{q}$, we let

$$
\mathbb{E}(J, \mathbb{I I}):=M \supseteq M_{1} \supseteq J M_{1} \supseteq J^{2} M_{1} \supseteq \cdots \supseteq J^{n-1} M_{1} \supseteq \ldots
$$

When there is no ambiguity we simply write $\mathbb{E}$ instead of $\mathbb{E}(J, \mathbb{M})$.

It is clear that this is a good $J$-filtration so that

$$
e_{0}(\mathbb{M})=e_{0}(\mathbb{E})=e_{0}(\mathbb{N}) .
$$

By considering the leading coefficients of $p_{\mathrm{MI}}^{1}(X)-p_{\mathrm{E}}^{1}(X)$ and $p_{\mathrm{E}}^{1}(X)-$ $-p_{\mathbb{N}}^{1}(X)$, since $J^{n+1} M \subseteq J^{n} M_{1} \subseteq M_{n+1}$ it follows that

$$
e_{1}(\mathbb{M}) \geq e_{1}(\mathbb{E}) \geq e_{1}(\mathbb{N}) \text {. }
$$

Proposition 2.7. Let $\mathrm{M}$ be a good q-filtration of the module $M$; if $\operatorname{dim} M=1$ and $J=($ a) with a $\mathbb{M}$-superficial for $\mathfrak{q}$, then

$$
e_{1}(\mathbb{E})-e_{1}(\mathbb{N}) \geq e_{0}(\mathbb{M})-h_{0}(\mathbb{M}) .
$$

PRoof. It is clear that for every $n \gg 0$ we have

$$
\begin{gathered}
p_{\mathbb{E}}^{1}(n)=\lambda\left(M / a^{n} M_{1}\right)=e_{0}(\mathbb{M})(n+1)-e_{1}(\mathbb{E}), \\
p_{\mathbb{N}}^{1}(n-1)=\lambda\left(M / a^{n} M\right)=e_{0}(\mathbb{M}) n-e_{1}(\mathbb{N}) .
\end{gathered}
$$

It follows that

$e_{1}(\mathbb{E})-e_{1}(\mathbb{N})=e_{0}(\mathbb{M})+\lambda\left(M / a^{n} M\right)-\lambda\left(M / a^{n} M_{1}\right) \geq e_{0}(\mathbb{M})-\lambda\left(a^{n} M / a^{n} M_{1}\right)$.

The conclusion follows because the map $M / M_{1} \stackrel{a^{n}}{\rightarrow} a^{n} M / a^{n} M_{1}$ is surjective.

We come now to the higher dimensional case; a natural strategy will be to lower dimension by using superficial elements.

The following Lemma is the key for doing the job. It is due to David Conti. 
Lemma 2.8. Let $M_{1}, \ldots, M_{r}$ be A-modules of dimension $d, J$ a m-primary ideal of $A$ and $\mathbb{M}_{1}=\left\{M_{1, j}\right\}, \ldots, \mathbb{M}_{r}=\left\{M_{r, j}\right\}$ good J-filtrations of $M_{1}, \ldots, M_{r}$ respectively. Then we can find elements $a_{1}, \ldots, a_{d}$ which are $\mathbb{M}_{i}$-superficial for $J$ for every $i=1, \ldots, r$.

If $d \geq 2$ and $M_{1}=\cdots=M_{r}=M$, then for every $1 \leq i \leq j \leq r$ we have

$$
e_{1}\left(\mathbb{M}_{i}\right)-e_{1}\left(\mathbb{M}_{i} / a_{1} M\right)=e_{1}\left(\mathbb{M}_{j}\right)-e_{1}\left(\mathbb{M}_{j} / a_{1} M\right) \text {. }
$$

Proof. Let

$$
\bigoplus_{i=1}^{r} \mathbb{M}_{i}:=\bigoplus_{i=1}^{r} M_{i} \supseteq \bigoplus_{i=1}^{r} M_{i, 1} \supseteq \bigoplus_{i=1}^{r} M_{i, 2} \supseteq \cdots \supseteq \bigoplus_{i=1}^{r} M_{i, j} \supseteq \cdots
$$

It is clear that this is a good $J$-filtration on $\bigoplus_{i=1}^{r} M_{i}$. Let us choose a $\bigoplus_{i=1}^{r} \mathbb{M}_{i^{-}}$ superficial sequence $\left\{a_{1}, \ldots, a_{d}\right\}$ for $J$. Then it is easy to see that $\left\{a_{1}, \ldots, a_{d}\right\}$ is a sequence of $M_{i}$-superficial elements for $J$ for every $i=1, \ldots, r$. This proves the first assertion. As for the second one, by (7) and (8) we have

$$
e_{1}\left(\mathbb{M}_{i}\right)-e_{1}\left(\mathbb{M}_{i} / a_{1} M\right)=\left\{\begin{array}{cl}
\lambda\left(0:_{M} a_{1}\right) & \text { if } d=2 \\
0 & \text { if } d \geq 3
\end{array}\right.
$$

from which the conclusion follows.

We first extend to the higher dimensional case the result of Proposition 2.7. We will see that the following result is a strengthened version of the classical inequality due to Northcott.

Proposition 2.9. Let $\mathbb{M}$ be a good $\mathfrak{q}$-filtration of the module $M$ and let $J$ be the ideal generated by a maximal sequence of M-superficial elements for $\mathfrak{q}$. Then we have

$$
e_{1}(\mathbb{E})-e_{1}(\mathbb{N}) \geq e_{0}(\mathbb{N})-h_{0}(\mathbb{M}) .
$$

Proof. If $\operatorname{dim} M=1$ we use Proposition 2.7. Let $\operatorname{dim} M \geq 2$; by the above Lemma we can find elements $a_{1}, \ldots, a_{d} \in J$ which are superficial for $J$ with respect to $\mathbb{E}$ and $\mathbb{N}$ at the same time. Further

$$
e_{1}(\mathbb{E})-e_{1}\left(\mathbb{E} / a_{1} M\right)=e_{1}(\mathbb{N})-e_{1}\left(\mathbb{N} / a_{1} M\right)
$$

and $J=\left(a_{1}, \ldots, a_{d}\right)$ by $(3)$.

The module $M / a_{1} M$ has dimension $d-1$ and $\mathbb{N} / a_{1} M$ is a good $J$-filtration on it. Further $a_{2}, \ldots, a_{d}$ is a maximal $\mathbb{N} / a_{1} M$-superficial sequence for $J$. If we let $K:=\left(a_{2}, \ldots, a_{d}\right)$, then the $K$-adic filtration on $M / a_{1} M$ is 
given by

$$
\left\{K^{j}\left(M / a_{1} M\right)\right\}_{j \geq 0}=\left\{\left(K^{j} M+a_{1} M\right) / a_{1} M\right\}_{j \geq 0}=\left\{\left(J^{j} M+a_{1} M\right) / a_{1} M\right\}_{j \geq 0}
$$

and thus coincides with $\mathbb{N} / a_{1} M$. On the other hand the filtration $\mathbb{E}\left(K, \mathbb{M} / a_{1} M\right):=M / a_{1} M \supseteq \frac{M_{1}+a_{1} M}{a_{1} M} \supseteq K \frac{M_{1}+a_{1} M}{a_{1} M} \supseteq K^{2} \frac{M_{1}+a_{1} M}{a_{1} M} \supseteq \ldots$ coincides with the filtration $\mathbb{E} / a_{1} M$ because $K^{j} \frac{M_{1}+a_{1} M}{a_{1} M}=\frac{J^{j} M_{1}+a_{1} M}{a_{1} M}$.
By induction we get

$$
\begin{aligned}
e_{1}(\mathbb{E})-e_{1}(\mathbb{N})=e_{1}\left(\mathbb{E} / a_{1} M\right) & -e_{1}\left(\mathbb{N} / a_{1} M\right) \\
& \geq e_{0}\left(\mathbb{N} / a_{1} M\right)-h_{0}\left(\mathbb{M} / a_{1} M\right)=e_{0}(\mathbb{M})-h_{0}(\mathbb{N})
\end{aligned}
$$

as desired.

Since $e_{1}(\mathbb{M}) \geq e_{1}(\mathbb{E})$ we obtain Nothcott's inequality which had been extended to non Cohen-Macaulay case by Goto and Nishida in [5].

CoRollary 2.10. Let $\mathbb{M}$ be a good $\mathfrak{q}$-filtration of the module $M$ and let $J$ be the ideal generated by a maximal sequence of $\mathbb{N}_{\mathbb{I}-\text { superficial }}$ elements for $\mathfrak{q}$. Then we have

$$
e_{1}(\mathbb{M})-e_{1}(\mathbb{N}) \geq e_{0}(\mathbb{M})-h_{0}(\mathbb{M}) .
$$

Given a good q-filtration $\mathbb{M}=\left\{M_{j}\right\}_{j \geq 0}$ of the $A$-module $M$ and an ideal $J$ generated by a maximal sequence of $M I$-superficial elements for $\mathfrak{q}$, we let for every $j \geq 0$ :

$$
v_{j}(\mathbb{M}):=\lambda\left(M_{j+1} / J M_{j}\right) .
$$

THEOREM 2.11. Let M be a good $\mathfrak{a}$-filtration of a module $M$ of dimension $d \geq 1, J$ an ideal generated by a maximal sequence of $\mathbb{N}-$-superficial elements for $\mathfrak{q}$ and $\mathbb{N}$ the $J$-adic filtration of $M$. Then we have

$$
e_{1}(\mathbb{M})-e_{1}(\mathbb{N}) \leq \sum_{j \geq 0} v_{j}(\mathbb{M}) .
$$

Proof. We prove the Theorem by induction on $d$. If $d=1$ we can apply Proposition 2.6; hence let $d \geq 2$. As in the above Proposition, we can find a system of generators $a_{1}, \ldots, a_{d}$ of $J$ with the properties that are superficial for $J$ with respect to $\mathbb{N}$ and $\mathbb{N}$, and verify

$$
e_{1}(\mathbb{N})-e_{1}\left(\mathbb{N} / a_{1} M\right)=e_{1}(\mathbb{M})-e_{1}\left(\mathbb{N} / a_{1} M\right)
$$


by (3). Also, if we let $K:=\left(a_{2}, \ldots, a_{d}\right)$, then $K$ is generated by a maximal $\mathbb{N} / a_{1} M$-superficial sequence for $J$ and the $K$-adic filtration on $M / a_{1} M$ is $\mathbb{N} / a_{1} M$. Thus, by induction, we get

$$
\begin{aligned}
e_{1}(\mathbb{N})-e_{1}(\mathbb{N}) & =e_{1}\left(\mathbb{N} / a_{1} M\right)-e_{1}\left(\mathbb{N} / a_{1} M\right) \leq \sum_{j \geq 0} v_{j}\left(\mathbb{N} / a_{1} M\right) \\
& =\sum_{j \geq 0} \lambda\left[\left(M_{j+1}+a_{1} M\right) /\left(K M_{j}+a_{1} M\right)\right] \\
& =\sum_{j \geq 0} \lambda\left[\left(M_{j+1}+a_{1} M\right) /\left(J M_{j}+a_{1} M\right)\right] \\
& =\sum_{j \geq 0} \lambda\left[M_{j+1} /\left(J M_{j}+\left(a_{1} M \cap M_{j+1}\right)\right)\right] \\
& \leq \sum_{j \geq 0} \lambda\left(M_{j+1} / J M_{j}\right) .
\end{aligned}
$$

We would like to study when the equality in the above Theorem holds. In the case $M$ is Cohen-Macaulay, we have a solution for a general filtration which broadly extends previous results of [7] and [6].

THEOREM 2.12. Let $\mathbb{M}=\left\{M_{j}\right\}_{j \geq 0}$ be a good $\mathfrak{q}$-filtration of the CohenMacaulay module $M$ of dimension $d \geq 1$ and $J$ an ideal generated by a maximal sequence of $\mathbb{M}$-superficial elements for $q$. Then we have

a) $e_{1}(\mathbb{N}) \leq \sum_{j \geq 0} v_{j}(\mathbb{M})$

b) $e_{2}(\mathbb{M}) \leq \sum_{j \geq 0} j v_{j}(\mathbb{M})$.

c) The following conditions are equivalent

1. depth $g r_{\mathbb{M}}(M) \geq d-1$.

2. $e_{i}(\mathbb{M})=\sum_{j \geq i-1}\left(\begin{array}{c}j \\ i-1\end{array}\right) v_{j}(\mathbb{M})$ for every $i \geq 1$.

3. $e_{1}(\mathbb{N})=\sum_{j \geq 0} v_{j}(\mathbb{M})$.

4. $e_{2}(\mathbb{M})=\sum_{j \geq 0} j v_{j}(\mathbb{M})$.

5. $P_{\mathrm{MI}}(z)=\frac{\lambda\left(M / M_{1}\right)+\sum_{j \geq 0}\left(v_{j}(\mathbb{M})-v_{j+1}(\mathbb{M})\right) z^{j+1}}{(1-z)^{d}}$.

Proof. Let $J=\left(a_{1}, \cdots, a_{d}\right)$ and $K=\left(a_{1}, \cdots, a_{d-1}\right)$; we first remark that, by Valabrega-Valla, depth $g r_{\mathbb{M}}(M) \geq d-1$ if and only if 
$M_{j+1} \cap K M=K M_{j}$ for every $j \geq 0$. Further we have

$$
v_{j}(\mathbb{M})=v_{j}(\mathbb{M} / K M)+\lambda\left(M_{j+1} \cap K M+J M_{j} / J M_{j}\right),
$$

hence $v_{j}(\mathbb{M}) \geq v_{j}(\mathbb{M} / K M)$ and equality holds if and only if $M_{j+1} \cap$ $\cap K M \subseteq J M_{j}$. This is certainly the case when $M_{j+1} \cap K M=K M_{j}$; hence, if depth $g r_{\mathbb{M}}(M) \geq d-1$, then $v_{j}(\mathbb{M})=v_{j}(\mathbb{M} / K M)$ for every $j \geq 0$. By induction on $j$, we can prove that the converse holds. Namely $M_{1} \cap K M=K M$ and, if $j \geq 1$, then we have

$$
\begin{aligned}
M_{j+1} \cap K M & \subseteq J M_{j} \cap K M=\left(K M_{j}+a_{d} M_{j}\right) \cap K M \\
& =K M_{j}+\left(a_{d} M_{j} \cap K M\right) \subseteq K M_{j}+a_{d}\left(M_{j} \cap K M\right) \\
& =K M_{j}+a_{d} K M_{j-1}=K M_{j}
\end{aligned}
$$

where $a_{d} M_{j} \cap K M \subseteq a_{d}\left(M_{j} \cap K M\right)$ because $a_{d}$ is regular modulo $K M$, while $M_{j} \cap K M=K M_{j-1}$ follows by induction.

Since $M / K M$ is Cohen-Macaulay of dimension one, we get

$$
e_{1}(\mathbb{M})=e_{1}(\mathbb{M} / K M)=\sum_{j \geq 0} v_{j}(\mathbb{M} / K M) \leq \sum_{j \geq 0} v_{j}(\mathbb{M}) .
$$

Equality holds if and only if depth $g r_{\mathrm{M}}(M) \geq d-1$. This, once more, proves a) and moreover gives the equivalence between 1 and 3 in c). By using Lemma 2.2 this also gives the equivalence between 1 . and 5 . in c).

Further, if $\mathfrak{a}$ is the ideal generated by $a_{1}, \cdots, a_{d-2}$, then, as before, we get

$$
e_{2}(\mathbb{M})=e_{2}(\mathbb{M} / \mathfrak{a} M) \leq e_{2}(\mathbb{M} / K M)=\sum_{j \geq 1} j v_{j}(\mathbb{M} / K M) \leq \sum_{j \geq 1} j v_{j}(\mathbb{M}) .
$$

This proves b) and $4 \Longrightarrow 1$. To complete the proof of the Theorem, we need only to show that $1 \Longrightarrow 2$. If depth $g r_{\mathbb{M}}(M) \geq d-1$, then $\mathbb{M}$ and $\mathbb{M} / K M$ have the same $h$-polynomial; this implies that for every $i \geq 1$ we have

$$
e_{i}(\mathbb{M})=e_{i}(\mathbb{M} / K M)=\sum_{j \geq i-1}\left(\begin{array}{c}
j \\
i-1
\end{array}\right) v_{j}(\mathbb{M} / K M)=\sum_{j \geq i-1}\left(\begin{array}{c}
j \\
i-1
\end{array}\right) v_{j}(\mathbb{M}) .
$$

If we do not assume that $M$ is Cohen-Macaulay, we are able to handle the problem only for the m-adic filtration on $A$.

THeOREM 2.13. Let $(A, \mathfrak{m})$ be a local ring of dimension $d \geq 1$ and $J$ the ideal generated by a maximal m-superficial sequence. The following 
conditions are equivalent:

1. $e_{1}(\mathfrak{m})-e_{1}(J)=\sum_{j \geq 0} v_{j}(\mathfrak{m})$.

2. $A$ is Cohen-Macaulay and depth $g r_{\mathfrak{m}}(A) \geq d-1$.

Proof. If $A$ is Cohen-Macaulay, then $e_{1}(J)=0$ and, by the above result, we get that 2) implies 1).

We prove now that 1) implies 2) by induction on $d$. If $d=1$, the result follows by Proposition 2.6 since $W \subseteq \mathfrak{m}$. Let $d \geq 2$; by Lemma 2.8 we can find a minimal basis $\left\{a_{1}, \ldots, a_{d}\right\}$ of $J$ such that $a_{1}$ is $J$-superficial, $\left\{a_{1}, \ldots, a_{d}\right\}$ is an $\mathfrak{m}$-superficial sequence and $e_{1}(\mathfrak{m})-e_{1}(J)=$ $=e_{1}\left(\mathfrak{m} /\left(a_{1}\right)\right)-e_{1}\left(J /\left(a_{1}\right)\right)$. Now $A /\left(a_{1}\right)$ is a local ring of dimension $d-1$ and $J /\left(a_{1}\right)$ is generated by a maximal $\mathfrak{m} /\left(a_{1}\right)$-superficial sequence. We can then apply Theorem 2.11 to get

$\sum_{j \geq 0} v_{j}(\mathfrak{m})=e_{1}(\mathfrak{m})-e_{1}(J)=e_{1}\left(\mathfrak{m} /\left(a_{1}\right)\right)-e_{1}\left(J /\left(a_{1}\right)\right) \leq \sum_{j \geq 0} v_{j}\left(\mathfrak{m} /\left(a_{1}\right)\right) \leq \sum_{j \geq 0} v_{j}(\mathfrak{m})$.

This implies

$$
e_{1}\left(\mathfrak{m} /\left(a_{1}\right)\right)-e_{1}\left(J /\left(a_{1}\right)\right)=\sum_{j \geq 0} v_{j}\left(\mathfrak{m} /\left(a_{1}\right)\right)
$$

which, by the inductive assumption, implies $A /\left(a_{1}\right)$ is Cohen-Macaulay. By Lemma $2.1 A$ is Cohen-Macaulay so that $e_{1}(J)=0$ and then $e_{1}(\mathfrak{m})=\sum_{j \geq 0} v_{j}(\mathfrak{m})$; this implies depth $g r_{\mathfrak{m}}(A) \geq d-1$ and the Theorem is
proved.

As a trivial consequence of the above result we have the following

Corollary 2.14. Let $(A, \mathfrak{m})$ be a local ring of dimension $d \geq 1, J$ the ideal generated by a maximal $\mathfrak{m}$-superficial sequence. If $e_{1}(J) \leq 0$, then

$$
e_{1}(\mathfrak{m}) \leq \sum_{j \geq 0} v_{j}(\mathfrak{m})
$$

Moreover, the following conditions are equivalent:

1. $e_{1}(\mathfrak{m})=\sum_{j \geq 0} v_{j}(\mathfrak{m})$.

2. $A$ is Cohen-Macaulay and depth $g r_{\mathfrak{m}}(A) \geq d-1$.

We notice that the condition $e_{1}(J) \leq 0$ is satisfied if, for example, $A$ is Buchsbaum, see [13] Proposition 2.7. It is verified as well if depth $A \geq d-1$; namely, if this is the case, given a $J$-superficial sequence 
$a_{1}, \ldots, a_{d-1}$, we have $e_{1}(J)=e_{1}\left(J /\left(a_{1}, \ldots, a_{d-1}\right)\right)$. Since $A /\left(a_{1}, \ldots, a_{d-1}\right)$ is one dimensional, by Lemma 2.4 we get $e_{1}\left(J /\left(a_{1}, \ldots, a_{d-1}\right)\right) \leq 0$. More in general Vasconcelos conjectured in [17] that $e_{1}(J)<0$ whenever $A$ is not Cohen-Macaulay. This is proved for local integral domain essentially of finite type over a field.

\section{Application to the Sally module.}

Given a primary ideal $\mathfrak{q}$ in the local ring $(A, \mathfrak{m})$ and a minimal reduction $J$ of $\mathfrak{q}$, Vasconcelos introduced in [17] the so-called Sally's module $S_{J}(\mathfrak{q})$ of $\mathfrak{q}$ with respect to $J$. It is defined by the exact sequence

$$
0 \rightarrow \mathfrak{q} A[J t] \rightarrow \mathfrak{q} A[\mathfrak{q} t] \rightarrow S_{J}(\mathfrak{q})=\bigoplus_{n \geq 1} \mathfrak{q}^{n+1} / J^{n} \mathfrak{q} \rightarrow 0 .
$$

We know that, if different from zero, $S_{J}(\mathfrak{q})$ is an $A[J t]$-module of the same dimension $d$ as $A$.

The Hilbert function of this graded module is

$$
H_{S_{J}(\mathfrak{q})}(n):=\lambda\left(q^{n+1} / J^{n} \mathfrak{q}\right),
$$

and its Hilbert series is

$$
P_{S_{J}(\mathfrak{q})}(z)=\sum_{n \geq 1} \lambda\left(q^{n+1} / J^{n} \mathfrak{q}\right) z^{n}
$$

We write $e_{i}\left(S_{J}(\mathfrak{q})\right)$ for the corresponding Hilbert coefficients.

In [19] it has been proved that if $A$ is Cohen-Macaulay then

$$
e_{0}\left(S_{J}(\mathfrak{q})\right) \leq \sum_{j \geq 1} \lambda\left(\mathfrak{q}^{j+1} / J \mathfrak{q}^{j}\right)
$$

and equality holds if and only if depth $g r_{\mathrm{q}}(A) \geq d-1$.

In this section we extend the definition of the Sally module to any good $q$-filtration of an $A$-module $M$ and prove that the inequality above holds in this generality and without the assumption that $M$ is Cohen-Macaulay. Further, we study when equality holds in the special case of the $q$-adic filtration.

Let $\mathrm{M}$ be a good q-filtration of the $A$-module $M$ of dimension $d$ and let $J$ be the ideal generated by a maximal $\mathbb{M}$-superficial sequence for $\mathfrak{q}$.

We let

$$
S_{J}(\mathbb{M}):=\bigoplus_{n \geq 1}\left(M_{n+1} / J^{n} M_{1}\right)
$$


and call it the Sally's module of $\mathbb{M}$ with respect to $J$. We remark that $S_{J}(\mathbb{M})$ is not a graded module associated to a filtration, but if we consider the filtration

$$
\mathbb{E}:=M \supseteq M_{1} \supseteq J M_{1} \supseteq J^{2} M_{1} \supseteq \ldots \supseteq J^{n} M_{1} \supseteq \ldots
$$

then $S_{J}(\mathbb{M})$ is related to $g r_{\mathrm{MI}}(M)$ and $g r_{\mathrm{E}}(M)$ by the following two short exact sequences:

$$
\begin{gathered}
0 \rightarrow J^{n-1} M_{1} / J^{n} M_{1} \rightarrow M_{n} / J^{n} M_{1} \rightarrow M_{n} / J^{n-1} M_{1} \rightarrow 0 \\
0 \rightarrow M_{n+1} / J^{n} M_{1} \rightarrow M_{n} / J^{n} M_{1} \rightarrow M_{n} / M_{n+1} \rightarrow 0 .
\end{gathered}
$$

Since $M_{n} / J^{n-1} M_{1}=\left(S_{J}(\mathbb{M})(-1)\right)_{n}$, by standard facts it follows that

$$
\operatorname{depth} g r_{\mathbb{M}}(M) \geq \min \left\{\operatorname{depth} S_{J}(\mathbb{M} I)-1 \text {, depth } g r_{\mathbb{E}}(M)\right\}
$$

Moreover we get

$$
P_{S_{J}(\mathbb{M})(-1)}(z)+P_{\mathbb{E}}(z)=P_{\mathrm{M}}(z)+P_{S_{J}(\mathrm{MI})}(z)
$$

so that

$$
(z-1) P_{S_{J}(\mathbb{M})}(z)=P_{\mathbb{M}}(z)-P_{\mathbb{E}}(z)
$$

If $\operatorname{dim} S_{J}(\mathbb{V})=d$, this implies that for every $i \geq 0$ we have

$$
e_{i}\left(S_{J}(\mathrm{MI})\right)=e_{i+1}(\mathrm{MI})-e_{i+1}(\mathbb{E})
$$

Now, by Proposition 2.9, we know that

$$
e_{1}(\mathbb{E})-e_{1}(\mathbb{N}) \geq e_{0}(\mathbb{M})-h_{0}(\mathbb{M}),
$$

so that

$$
e_{0}\left(S_{J}(\mathbb{M})\right)=e_{1}(\mathbb{M})-e_{1}(\mathbb{E}) \leq e_{1}(\mathbb{M})-e_{1}(\mathbb{N})-e_{0}(\mathbb{M})+h_{0}(\mathbb{M}) .
$$

Notice that, for the special case of the q-adic filtration on $A$, this result has been proved in [3].

Now we can use Theorem 2.11, to get

$$
e_{0}\left(S_{J}(\mathbb{M})\right) \leq \sum_{j \geq 0} v_{j}(\mathbb{M})-e_{0}(\mathbb{M})+h_{0}(\mathbb{M}) .
$$

Thus we have proved the following

Theorem 3.1. Let $\mathbb{M}$ be a good q-filtration of the A-module $M$ of dimension $d$ and let $J$ be the ideal generated by a maximal MI-superficial sequence for $\mathfrak{q}$. If $\operatorname{dim} S_{J}(\mathbb{V})=d$, then

$$
e_{0}\left(S_{J}(\mathrm{MI})\right) \leq \sum_{j \geq 0} v_{j}(\mathrm{MI})-e_{0}(\mathbb{M})+h_{0}(\mathbb{M}) .
$$


It is clear that if the equality holds above then $e_{1}(\mathbb{M})-e_{1}(\mathbb{N})=$ $=\sum_{j \geq 0} v_{j}(\mathrm{M})$. Hence, in the case of the m-adic filtration on $A$, we can apply Theorem 2.13 and we get that $A$ is Cohen-Macaulay and depth $g r_{\mathrm{m}}(A) \geq d-1$.

In this way we get the following result which completes Theorem 2.13.

THEOREM 3.2. Let $(A, \mathfrak{m})$ be a local ring of dimension $d \geq 1$ and $J$ the ideal generated by a maximal $\mathfrak{m}$-superficial sequence. If $\operatorname{dim} S_{J}(\mathfrak{m})=d$, then $e_{0}\left(S_{J}(\mathfrak{m})\right) \leq \sum_{j>0} v_{j}(\mathfrak{m})-e_{0}(\mathfrak{m})+1$. Moreover the following conditions are equivalent:

1. $e_{0}\left(S_{J}(\mathfrak{m})\right)=\sum_{j \geq 0} v_{j}(\mathfrak{m})-e_{0}(\mathfrak{m})+1$

2. $e_{1}(\mathfrak{m})-e_{1}(J)=\sum_{j \geq 0} v_{j}(\mathfrak{m})$

3. A is Cohen-Macaulay and depth $g r_{\mathfrak{m}}(A) \geq d-1$.

We finish the paper with the following Theorem which adds some more equivalent conditions, involving the Sally module of $M$, to those of Theorem 2.12 in the case $M$ is Cohen-Macaulay. The result extends to a considerable extent a series of results proved in [19] for the special case of the q-adic filtration on $A$.

First we remark that if $M$ is Cohen-Macaulay, then $g r_{\mathbb{E}}(M)$ is CohenMacaulay with minimal multiplicity and hence

$$
P_{\mathbb{E}}(z)=\frac{h_{0}(\mathbb{M})+\left(e_{0}(\mathbb{M})-h_{0}(\mathbb{M})\right) z}{(1-z)^{d}} .
$$

In particular $e_{1}(\mathbb{E})=e_{0}(\mathbb{M})-h_{0}(\mathbb{M})$.

By using (14), (12), (13) we get

1. If $\operatorname{dim} S_{J}(\mathbb{M})=d$, then $e_{0}\left(S_{J}(\mathbb{N})\right)=e_{1}(\mathbb{N})-e_{0}(\mathbb{M})+h_{0}(\mathbb{M})$

2. depth $g r_{\mathbb{M}}(M) \geq \operatorname{depth} S_{J}(\mathbb{M})-1$

3. $(z-1) P_{S_{J}(\mathbb{M})}(z)=P_{\mathbb{M}(}(z)-\frac{\lambda\left(M / M_{1}\right)+\left(e_{0}(\mathbb{M})-\left(\lambda\left(M / M_{1}\right)\right) z\right.}{(1-z)^{d}}$.

If $M$ is the q-adic filtration on $M$ then it is easy to see that the assumption $\operatorname{dim} S_{J}(\mathrm{M})=d$ is equivalent to $S_{J}(\mathbb{M}) \neq 0$. In fact, by (13), we have that $\operatorname{dim} S_{J}(\mathbb{M})=d$ if and only if $e_{1}(\mathbb{M})>e_{1}(\mathbb{E})=e_{0}(\mathbb{M})-h_{0}(\mathbb{M})$. This is equivalent to $M_{2} \neq J M_{1}$ and hence $S_{J}(\mathbb{M}) \neq 0$. 
THeorem 3.3. Let $M$ be a Cohen-Macaulay A-module of dimension $d \geq 1, \mathfrak{q}$ a primary ideal in $A, \mathbb{M}$ the $\mathfrak{q}$-adic filtration on $M$ and $J$ the ideal generated by a maximal sequence of M-superficial elements for $\mathfrak{q}$. The following conditions are equivalent:

1. $e_{0}\left(S_{J}(\mathbb{M})\right)=\sum_{j \geq 1} v_{j}(\mathbb{M})$

2. $P_{S_{J}(\mathbb{M I})}(z)=\frac{\sum_{j \geq 1} v_{j}(\mathbb{M}) z^{j}}{(1-z)^{r}}$

3. $S_{J}(\mathrm{MI})$ is Cohen-Macaulay

and each of them is equivalent to the equivalent conditions of Theorem 2.12 .

Proof. We have $e_{0}\left(S_{J}(\mathbb{M})\right)=e_{1}(\mathbb{M})-e_{0}(\mathbb{M})+h_{0}(\mathbb{M})$. Hence, by Theorem 2.12, we get

$$
e_{0}\left(S_{J}(\mathbb{M})\right) \leq \sum_{j \geq 0} v_{j}(\mathbb{M})-e_{0}(\mathbb{M})+h_{0}(\mathbb{M})=\sum_{j \geq 1} v_{j}(\mathbb{M}) .
$$

By Theorem 2.12, the equality holds if and only if $e_{1}(\mathrm{M})=\sum_{j \geq 0} v_{j}(\mathrm{M})$. Hence 1. is equivalent to 1., 2., 3., 4., 5. of Theorem 2.12. Because 1. is equivalent to

$$
P_{\mathbb{M}}(z)=\frac{\lambda\left(M / M_{1}\right)+\sum_{j \geq 0}\left(v_{j}(\mathbb{M})-v_{j+1}(\mathbb{M})\right) z^{j+1}}{(1-z)^{d}}
$$

and we know that

$$
(z-1) P_{S_{J}(\mathbb{M I})}(z)=P_{\mathrm{M}}(z)-\frac{\lambda\left(M / M_{1}\right)+\left(e_{0}(\mathbb{M})-\left(\lambda\left(M / M_{1}\right)\right) z\right.}{(1-z)^{d}},
$$

it is easy to see that 1 . is also equivalent to 2 . Now, since depth $g r_{\mathrm{M}}(M) \geq$ $\geq \operatorname{depth} S_{J}(\mathbb{M})-1$, we get that 3 . implies depth $g r_{\mathbb{M}}(M) \geq d-1$ which is equivalent to 1 .

We have only to prove that 2. implies 3. We may assume $S_{J}(\mathrm{M})$ of dimension $d$ and recall that $S_{J}(\mathbb{M})$ is a $\mathbb{R}(J)=A[J T]$-module and we have $S_{J}(\mathbb{M}) / J T S_{J}(\mathbb{M})=\bigoplus_{n \geq 1} M_{n+1} / J M_{n}$. By 2 . we deduce that $P_{S_{J}(\mathbb{M})}(z)=$ $=\frac{1}{(1-z)^{d}} P_{\left.S_{J}(\mathbb{N}) / J T S_{J}(\mathbb{N})\right)}(z)$. Then $J T$ is generated by a regular sequence of lenght $d=\operatorname{dim} S_{J}(\mathbb{M})$ and hence $S_{J}(\mathbb{M})$ is Cohen-Macaulay. 


\section{REFERENCES}

[1] M.F. АтніYah - I.G. Macdonald, Introduction to Commutative Algebra, Addison-Wesley (1969).

[2] W. Bruns - J. Herzog, Cohen-Macaylay rings, Revised edition, Cambridge Studies in Advanced Mathematics, 39 (Cambridge University Press, Cambridge, 1998).

[3] A. Conso, Sally modules of m-primary ideals in local rings, arXiv:math/ 0309027v1 [math.AC].

[4] J. Elias - G. VAlla, Rigid Hilbert functions, J. Pure and Applied Algebra, 71 (1991), pp. 19-41.

[5] S. Goto - K. NishidA, Hilbert coefficients and Buchsbaumness of the associated graded ring, J. Pure and Appl. Algebra, 181, no. 1 (2003), pp. 64-76.

[6] A. Guerrieri - M.E. Rossi, Hilbert coefficients of Hilbert filtrations, J. Algebra, 199, no. 1 (1998), pp. 40-61.

[7] S. Huckaba - T. Marley, Hilbert coefficients and the depths of associated graded rings, J. London Math. Soc., 56 (1997), pp. 64-76.

[8] C. Huneke - I. Swanson, Integral closure of Ideals, Rings and Modules, London Mathematical Lecture Notes, Series 336 (Cambridge University Press, 2006).

[9] C. Polini - B. Ulrich - W. Vasconcelos, Normalization of ideals and Briancon-Skoda numbers, Math. Research Letters, 12 (2005), pp. 827-842.

[10] M.E. Rossi - G. VAlla, Hilbert Functions of Filtered Modules, arXiv: 0710.2346v1 [math.AC].

[11] J.P. SERRE, Algebre local multiplicite, Lecture Notes in Math., 11 (SpringerVerlag, Berlin, 1965).

[12] B. SinGH, Effect of a permissible blowing-up on the local Hilbert functions, Invent. Math., 26 (1974), pp. 201-212.

[13] J. StÜCKRAD - W. VogeL, Buchsbaum rings and applications, (SpringerVerlag, Berlin, 1986).

[14] I. Swanson, A note on analytic spread, Comm. in Algebra, 22, No. 2 (1994), pp. 407-411.

[15] P. Valabrega - G. Valla, Form rings and regular sequences, Nagoya Math. J., 72 (1978), pp. 93-101.

[16] G. VALLA, On form rings which are Cohen-Macaulay, J. Algebra, 59 (1979), pp. 247-250.

[17] W. VAsconcelos, Hilbert functions, analytic spread and Koszul homology, Contemp. Math., 159 (1994), pp. 401-422.

[18] W. Vasconcelos, The Chern numbers of local rings, Michigan Math. J., Vol. 57 (2008), pp. 725-743

[19] M. VAz Pinto, Hilbert Functions and Sally modules, J. Algebra, 192 (1997), pp. 504-523.

Manoscritto pervenuto in redazione il 5 febbraio 2008. 